\title{
Reading Out the Synaptic Echoes of Low-Level Perception in V1
}

\author{
Yves Frégnac \\ UNIC-CNRS, Gif-sur-Yvette, France \\ fregnac@unic.cnrs-gif.fr \\ http://www.unic.cnrs-gif.fr
}

\begin{abstract}
Primary visual cortex (V1) in the mammalian brain computes on the fly perceptual primitives (form, motion, visual flow) from the feedforward bombardment of retinal events channeled through the thalamus. At the same time, it integrates the distributed feedback of higher cortical areas involved in more elaborate cognitive functions. The reverberating activity evoked by the interplay between these two streams has been hypothesized to form the trace of the low-level computational operations written on the "high resolution buffer" of primary cortical areas [1]. In vivo intracellular electrophysiology in V1 offers the unique possibility of listening to the synaptic echoes of the effective perceptual network at work. On the basis of the comparison between functional synaptic imaging and voltage sensitive dye imaging, I will show that the emergence of macroscopic features of perception (Gestalt and motion flow related percepts) in early sensory cortical areas can be predicted from the read-out of analog graded events (synaptic potentials) operating at a more microscopic integration level.
\end{abstract}

The field of neuromorphic computation has grown from the idea that inspiration for future computational architectures can be gained from a better understanding of information processing in biological neural networks. An illustration of the impact of Biology in Artificial Vision and Image Processing is given by studies of the early visual system in the mammalian brain. Information coding of the sensory world in our brain is both digital, in terms of spike-based events, and analog, in terms of slower, subthreshold changes in membrane voltage resulting from the ongoing or evoked barrage of synaptic inputs. In vivo intracellular electrophysiology during sensory processing in primary cortical areas offers the unique possibility of listening to the "synaptic rumour" of the effective network at work, extracting primitives of our environment (local contrast, form, retinal flow, ..). The analysis of synaptic echoes captured by the recording electrode in a single V1 cell allows to infer, indirectly in cortical space, the dynamics of the effective input network afferent to the recorded cell.

We have applied this reverse engineering method to demonstrate the propagation of visually evoked activity through lateral (and possibly feedback) connectivity in the primary cortex of higher mammals. This approach, based on functional synaptic imaging at the microscopic integration level, is compared here with a macroscopic imaging technique, based on the use of voltage sensitive fluorescent dyes. The conceptual 
novelty of this multi-scale imaging approach is to explore to what extent emerging macroscopic features of low level (non attentive) perception in early sensory cortical areas (V1, V2, V4, MT), related to motion flow and form processing and Gestalt psychological laws, can be predicted from more microscopic levels of neural integration (conductance and membrane potential dynamics, synaptic receptive fields).

\section{A Topological Paradox in the Early Visual System}

The biological foundations of low-level visual perception in the mammalian brain show an apparent paradox:

On the one hand, the functional specificity of the early visual system underlying non-attentive perception seems to be best explained by a parallel cascade of serial filters from retina to cortex [2]. The synaptic impact of this topographic feed-forward projection is strong and results in multiple ordered point-to-point representations of the retinal periphery onto central target neural structures: most thalamic and cortical neurons express in their discharge a "tubular" view of the visual world. Spiking responses are evoked only when visual stimuli are presented within a small retinal window, defined as the "minimal discharge field".

On the other hand, the anatomical architecture along the visual thalamo-cortical pathway includes a profusion of feedback routes, from cortex to thalamus and from higher-order processing stations to primary visual cortex (V1), as well as intrinsic lateral and recurrent connections confined within each processing relay stage [3].

Thus, the functional binding of distant points in the primary cortical representation by lateral and feedback connectivity introduces a mismatch with the retinotopic order imposed by the feedforward projections. How this topological conflict is solved by the early visual system is still poorly understood (review in [4]).

\section{New Methodologies in Multi-scale Brain Imaging}

In order to visualize the functional influence of lateral connections, experimental approaches, more invasive methods than fMRI, allow to detect subthreshold neural activation in addition to evoked spike activity. One method is to monitor the spread of evoked activation relayed across the superficial cortical layers, the representation plane of the retinal space, using the voltage-sensitive dye (VSD) imaging [5]: CCD cameras reach a spatial $(<50 \mathrm{~mm})$ and temporal precision $(<1 \mathrm{~ms})$ compatible with the structural columnar scale and the time-course of synaptic responses. The most adequate stimuli (oriented luminance gratings) are optimized to fire cortical columns sharing the same orientation preference, and can be limited (focal) or not (full field) to visual cortical receptive fields extent [6]. Such techniques provide the macroscopic imaging of functional networks across areas V1 and V2 in rodents, ferrets, cats and monkeys.

Rather than looking at the global evoked dynamics of the network, a complementary approach is to address the microscopic organization scale and focus on the synaptic bombardment of a single neuron. Intracellular electrophysiology with sharp 
electrodes can be used to continuously monitor the membrane potential of a single neuron for several hours, even in vivo [7,8]. The recorded irregular asynchronous spiking activity is the result of the transient but repeated convergence arising from multiple synaptic sources in the network. For the past 25 years, my lab has been developing a reverse engineering approach, based on demultiplexing the composite rumour of synaptic echoes recorded in a single cell. This new method, called "Functional synaptic imaging" [7], considers cortex as a chamber of echoes produced by visual activation. Its principle is similar to that of echography in the etymological sense (transcription of echoes) and equivalent to the principle of time reversal mirrors in acoustic physics and medicine [9]. Its validity relies however on the assumption that the input sources are separable in space and their synaptic influence travels in time with similar speed. This condition is rarely met in the general case, but seems to be valid for sparse stimulation regimes or during ongoing activity. Thus, functional synaptic imaging gives a prediction of the macroscopic activation of the network in space and time, which can be confronted with the direct observation of the spatiotemporal cortical dynamics evoked in the superficial layers of cortex, using voltagesensitive dyes $[6,10]$.

\section{Predicting Travelling Waves from Synaptic Echoes}

The hypothesis of a travelling wave [7] was made initially on the assumption of symmetry in exuberant intrinsic connectivity: since V1 is a highly recurrent network, we assume that each cell is connected reciprocally to any other cell, with identical propagation delays from and to. This theoretical shortcut allows the inference of propagation patterns (the cell being seen as a "wave emitter") solely on the basis of the spatio-temporal maps of stimulus-locked synaptic responses recorded in a single cell (the cell being seen as an "echo receiver"). This suggests that the information received from the RF center in the cortex through the feedforward afferents is then propagated radially by the horizontal connectivity to neighboring regions of the visual cortex over a distance that may correspond to up to 10 degrees of visual angle. These data led successfully to the functional identification and reconstruction of a propagating wave of visual activity relayed by the horizontal connectivity.

The principle of calculation of the propagation speed of the intracortical "horizontal" wave is straightforward when comparing the synaptic effects of two elementary stimuli (white bar), one in the core of the minimal discharge field, the other in the "silent" surround. The distance between the primary points of the feedforward impact produced in cortex by the two stimuli can be predicted on the basis of their relative retinal eccentricity $\Delta \mathrm{x}_{\mathrm{v}}$ and the value of the retino-cortical magnification factor (RCMF). This factor can be measured electrophysiologically ([11] in cat), by 2deoxyglucose metabolic labeling ([12] in monkey), by intrinsic imaging ([13] in mouse and [14] in ferret) and by fMRI ([15] in humans). Thus, beyond a certain scale of spatial integration (larger than the columnar grain), any distance in visual space $\Delta \mathrm{x}_{\mathrm{v}}$ can be converted to a distance in visual cortex $\Delta \mathrm{x}_{\mathrm{c}}$. The spatial range of the subthreshold field extent agrees with the anatomical description of 4-7 mm horizontal axons running across superficial layers [16]. Furthermore, the electrophysiological 
recordings give access to the delay $\Delta \mathrm{t}_{\mathrm{c}}$ between the two synaptic echoes obtained through the feed-forward and the horizontally-mediated pathways. By dividing the inferred cortical distance $\Delta \mathrm{x}_{\mathrm{c}}$ in cortex by the recorded delay $\Delta \mathrm{t}_{\mathrm{c}}$, an apparent horizontal propagation speed can be computed within the cortical map, hence in the plane of the layers of V1. This rough estimate assumes a linear transformation between retinal and cortical coordinates, which is not the case in the human visual system, but, in the cat, the non-linear logpolar transformation [17] has a reduced impact in the representation domain of the $5^{\circ}$ around area centralis. The propagation speeds we inferred with or without logpolar correction range between 0.02 and $2 \mathrm{~m} / \mathrm{s}$, with a peak between 0.1 and $0.4 \mathrm{~m} / \mathrm{s}$.

These velocity values have since been confirmed for other sensory cortical structures, such as somatosensory cortex [18]. They are thus ten times slower than X-type thalamic input and feedback propagation from higher cortical areas (2 $\mathrm{m} / \mathrm{s}$ in [19]) and one hundred times slower than the fast Y-pathway $(8-40 \mathrm{~m} / \mathrm{s}$ in [20]). They are in fact within the order of magnitude of conduction speeds measured in vitro and in vivo along non-myelinated horizontal cortical axon fibers [21]. Recent reports based on cortical LFPs triggered on LGN spike activity rule out the possibility that divergence of LGN axons may also contribute to the build-up of the observed latency shifts (see for instance [22]).

\section{Visualizing Travelling Waves with VSD Network Imaging}

The macroscopic reconstruction of intra-V1 waves on the basis of microscopic echoes, described in the previous section, remains however an extrapolation made between two scales of spatial organization differing by two orders of magnitude (neuron vs map). The VSD technique, particularly adapted to layered structures, takes advantage of the fast changes in the fluorescence by the dye as a function of the state of depolarization of the membrane in which it is incorporated. It gives an unprecedented view of the state of dendritic tuft membrane depolarization in the superficial layers of the cortex, with a time sensitivity close to that of intracellular recordings $[5,23,24]$. Since their pioneering study of cortical spread function [5], the team of Amiram Grinvald has provided detailed quantifications of intracortical dynamics evoked by various inducer stimuli (square, annular grating) and documented the slow propagation of an activation wavefront, in area 17 or 18 , consistent in speed with the conduction velocity of horizontal connections $[6,26]$.

Many groups have since confirmed the propagation of spontaneous and evoked waves across the cortical laminar planes in visual primary and secondary cortical areas of rodents [27], ferrets [28,29] and higher mammals [30]. The visually evoked waves exhibit sterotyped features and show invariance with the parameters of the drifting grating, such as orientation and temporal or spatial frequency. In a contrasted way, the "ongoing" (spontaneous) waves vary in kinetics. They do not respect area boundaries, and propagate without interruption throughout the entire imaged area (up to $4 \mathrm{~mm}$ ) more slowly than the evoked activity. It is likely that the two forms of propagating activity are generated by different mechanisms and that the slowest waves is a polysynaptic column-to column propagation of "up" states [31], similar to the "rolling waves » previously reported with calcium imaging in vitro [32]. 
In summary, these different imaging studies fully corroborate the prediction of a travelling wave across cortex, that we extracted some fifteen years ago from our intracellular recordings [7,8]: synaptic responses elicited by stimuli placed far from the center of the receptive field showed decreasing amplitude and increasing onset delay with the relative eccentricity. Most remarkably both types of imaging methods (microscopic or mesoscopic) give the same mean estimate of propagation speed $(0.10-0.40 \mathrm{~m} / \mathrm{s})$ although they are based on different measurements and analysis.

\section{Gestalt Principles as an Emerging Feature of Visual Cortical Dynamics}

One of the functional roles attributed to long-distance horizontal axons in visual cortex is to link columns sharing similar orientation preference [33]. However, the anatomical evidence in favor of such bias remains rather scarce in the cat cortex. Combinations of optical imaging and intracellular labeling show indeed a diversity of potential links established between orientation columns which do not obey, at least at the statistical significance level, the rule "who is alike tend to be coupled together". As a consequence, at a more integrated mesoscopic level, the net functional effect cannot be predicted. A recent collaborative work between our lab and the group of Amiram Grinvald, addressed this issue by combining VSD and functional synaptic imaging [6]. It showed unexpectedly that a local oriented stimulus evoked an orientation selective activity component which remained confined to the feed-forward cortical imprint of the stimulus (space constant $1 \mathrm{~mm}$ ). Thus, a single local stimulus does not propagate orientation preference through the long-range horizontal cortical connections. In contrast, when increasing spatial and temporal summation, both optical imaging and intracellular measurements showed the emergence of an iso-orientation selective spread. We conclude that stimulus-induced cooperativity is a necessary constraint for the emergence of iso-functional binding.

Such a contrasted and conditional behaviour may serve two functions: 1) for the local oriented stimulus, a non-selective divergent connectivity pattern may facilitate detection of high-order topological properties (e.g. orientation discontinuities, corners, geons). 2) summation of multiple oriented sources in the far "silent" surround can optimize the emergence of iso-orientation preference links. For instance, oriented annular stimuli may recruit iso-oriented sources collinearly organized with the orientation preference axis of the target column/cell; similar synergy may be obtained in the temporal domain when sources, independent of their exact location, share the same motion direction sensitivity as the target grating. Both of these configurations, which are confounded in annular aperture protocols, correspond to the neural implementation of the Gestalt's continuity and common fate principles [34,35].

This conclusion might explain why multiple stroke animations with oriented elements favor the propagation of perceptual biases such as observed during apparent motion illusions. We hypothesized from our own intracellular findings that the perception of speed could be differentially modulated during apparent motion sequences of oriented stimuli, either collinear and aligned with respect to the motion axis or at 
an angle to it [36]. We devised a series of psychophysical experiments in humans that aimed at testing the influence of orientation relative to the motion axis on perceived speed [37]. Observers were asked to discriminate during a forced choice task between the relative speeds of two apparent-motion (AM) sequences. Results showed the induction of a "speedup" illusion which was observed for retinal speeds compatible with intracortical horizontal wave propagation. For this range of speed (equivalent to saccadic displacement), a Gabor patch moving along its orientation axis appears much faster than a Gabor patch oriented at an angle to the motion axis.

\section{Apparent Motion}

\section{The Dynamic Association Field}
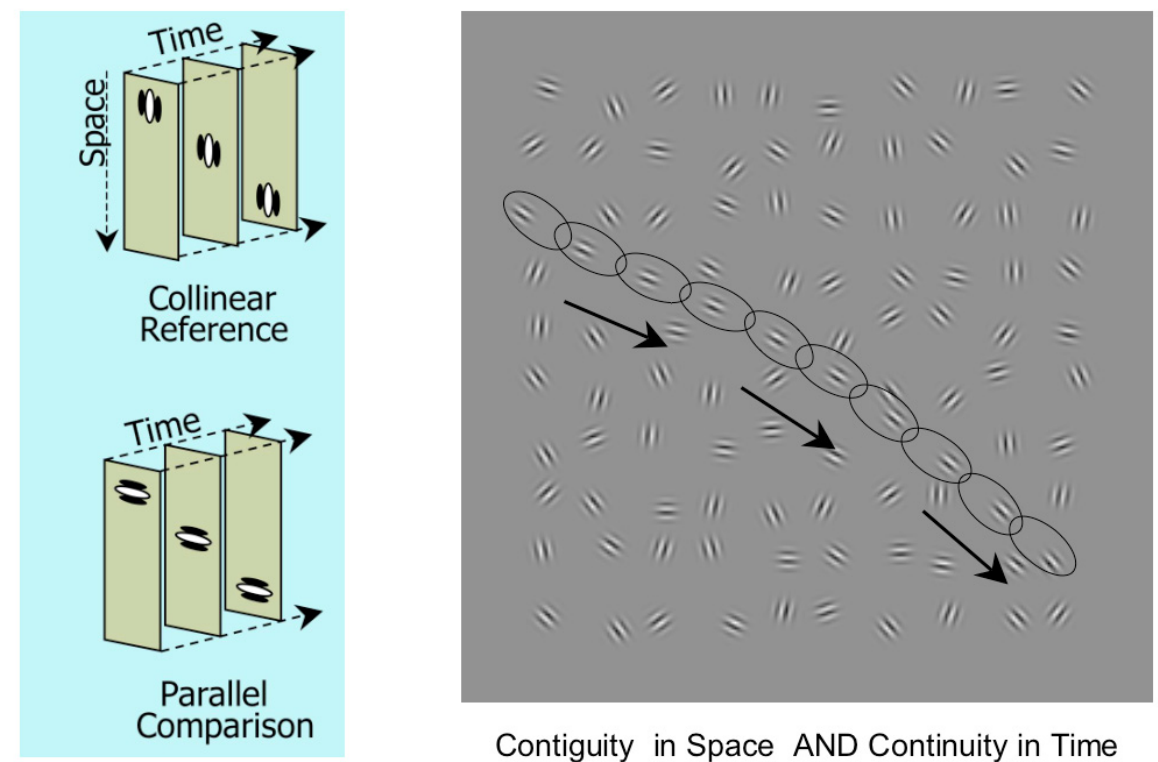

Contiguity in Space AND Continuity in Time

\section{Fig. 1. The "Dynamic Association Field" Hypothesis}

Left: two-forced choice apparent motion protocol, where human observers report which sequence of oriented elements is seen "faster" [36,37]. Two configurations were compared (collinear and parallel), in which the orientation of each element was respectively collinear or orthogonal to the motion axis. Human subjects perceive the "collinear" apparent motion (AM) configuration as being more than two times faster than the "parallel" one, when the feedforward input evoked by the three-stroke AM stimuli travels in V1 cortex at the speed of horizontal propagation ( 0.1 to $0.3 \mathrm{~m} / \mathrm{s})$. Right: the "dynamic association field" hypothesis; local oriented inputs (Gabor patches) induce a facilitation wave of activity travelling along horizontal connections intra V1. This wave binds in space and time proximal receptive fields with co-linear preferred orientations, thus creating a contiguous path of temporal integration. The associative strength of the perceptual effect is maximal when the asynchronous feedforward sequence produced by joint strokes of apparent motion (arrow) travels in phase in the cortical network with the visually evoked horizontal propagation. The retinal flow speed at which this effect predominates is compatible with retinal slip produced by saccadic eye-movements during active scanning of contours. 
These different experimental observations led us to formulate the concept of the "dynamic association field" [10], which adds a temporal coordination dimension to the static "association field" introduced originally by Hess and Field [38,39]. In its dynamic version, the revised concept assumes that local oriented inputs (Gabor patches) induce a facilitation wave of activity travelling along horizontal connections intra V1 (Figure 1, right panel). This coordination wave tends to bind proximal receptive fields with co-linear preferred orientations, thus creating a contiguous path of temporal integration. The associative strength of the perceptual effect is maximal when the asynchronous feedforward sequence produced by joint strokes of apparent motion travels in phase with the visually evoked horizontal intracortical propagation. Recent intracellular work from our lab shows that sparse apparent motion two-stroke noise appears as a powerful stimulus condition to trigger the coordination of synaptic activity along centripetal motion streaks attuned to the orientation preference of the target cells (Carelli, Pananceau, Gerard-Mercier, Baudot, Monier and Frégnac, unpublished).

\section{Conclusion: Propagation of Network Belief and Bayesian Models of Perception}

This comparative overview of synaptic and network imaging in visual primary cortex summarizes two important findings: 1) intracellular recordings can be used to detect at the single cell level the synaptic trace of waves of visual activation travelling along long-distance horizontal connections; 2) VSD imaging methods reveal the macroscopic propagation pattern predicted from these synaptic echoes. One obvious consequence is that the V1 network should not be considered as an ordered mosaic of independent "tubular" analyzers, but rather as a constellation of wide field integrators, integrating simultaneously input sources arising from much larger regions of visual space than previously thought. The collective behaviour of these integrators is coordinated during sensory processing by the anisotropic propagation of stimulusinduced facilitatory waves travelling at slow speed along unmyelinated axons within the superficial cortical layers. Thus, primary visual cortical neurons have the capacity to combine information originating from different points of the visual field, in a spatio-temporal reference frame centered on the discharge field itself. This ability imposes precise constraints in time and in space on the efficacy of the summation process of elementary synaptic responses, and could subserve the emergence of elementary self-organizing processes necessary for implementing Gestalt principles.

A likely role of horizontal propagation is to favor the build-up of coherent selforganized knowledge in the network, and broadcast it to distant parts of the network. The functional features of these slow waves support the hypothesis of promoting a "dynamic association" field, which facilitates the integration of feed-forward inputs yet to come. During perceptual illusions (apparent motion, line motion), these waves propagate the network belief of the possible presence of a global percept (the "whole": here, continuous motion of a space-invariant shape) before the illusory percept becomes validated by the sequential presentation of the "parts" (signaled by 
direct focal feed-forward waves). Such dynamics obey closely the Gestalt prediction that the emergence of the "whole" should precede in time the detection of the "parts".

These cortical processes result, at the perceptual level, in the propagation of functional biases (binding of collinear contour and iso-directional motion) which spreads beyond the scale of the cortical hyper column. It remains to be determined whether the correlations we report between perception and horizontal propagation result solely of neural processes intrinsic to V1, or whether they reflect the reverberation in V1 of collective feedback from multiple secondary cortical areas. It may be indeed envisioned that the primary visual cortex plays the role of a generalized echo chamber fed by other cortical areas (visual or not) which participate in the coding of shape and motion in space: accordingly, the waves travelling across V1 would signal the emergence of perceptual coherence when sufficient synergy is reached between the different cortical analyzers. A new challenge is launched at the interface between electrophysiology and brain imaging, where multiscale imaging approaches give unprecedented access to generic principles of binding between Form and Motion, which operate in the early visual system during low-level (non attentive) perception.

Acknowledgements. The reviewed work of the Lab was done in collaboration with Vincent Bringuier, Frédéric Chavane, Cyril Monier, and more recently with Pedro Carelli, Julien Fournier, Florian Gérard-Mercier, Olivier Marre and Marc Pananceau for intracellular physiology and VSD imaging and with Sébastien Georges, Jean Lorenceau and Peggy Series for psychophysics. The current work is supported by CNRS, and grants from ANR (NatStats and V1-complex) and the European Community FET-Bio-I3 programs (IP FP6: FACETS (015879), IP FP7: BRAINSCALES (269921) and Brain-i-nets (243914)).

\section{References}

1. Mumford, D.: On the computational architecture of the neocortex. II. The role of corticocortical loops. Biol. Cybern. 66, 241-251 (1992)

2. Carandini, M., Demb, J.B., Mante, V., Olshausen, R.A., Tolhurst, D.J., Dan, Y., Gallant, J.L., Rust, N.: Do we know what the early visual system does? J. Neurosci. 25, 10577-10597 (2005)

3. Gilbert, C., Das, A., Ito, M., Kapadia, M., Westheimer, G.: Spatial integration and cortical dynamics. Proc. Natl. Acad. Sci. USA 93, 615-622 (1996)

4. Séries, P., Lorenceau, J., Frégnac, Y.: The silent surround of V1 receptive fields: theory and experiments. Journal of Physiology 97(4-6), 453-474 (2003)

5. Grinvald, A., Hildesheim, R.: VSDI: a new era in functional imaging of cortical dynamics. Nat. Rev. Neurosci. 5(11), 874-885 (2004)

6. Chavane, F., Sharon, D., Jancke, D., Marre, O., Frégnac, Y., Grinvald, A.: Lateral spread of orientation selectivity in V1 is controlled by intracortical cooperativity. Front. System Neuroscience 5(4), 1-26 (2011), doi:10.3389/fnsys.2011.00004.

7. Frégnac, Y., Bringuier, V.: Spatio-temporal dynamics of synaptic integration in cat visual cortical receptive fields. In: Aertsen, A., Braitenberg, V. (eds.) Brain Theory: Biological Basis and Computational Theory of Vision, pp. 143-199. Springer, Amsterdam (1996) 
8. Bringuier, V., Chavane, F., Glaeser, L., Frégnac, Y.: Horizontal propagation of visual activity in the synaptic integration field of area 17 neurons. Science 283, 695-699 (1999)

9. Fink, M.M.: Time reversal in acoustics. Contemporary Physics 37, 95-109 (1996) 1366-5812

10. Frégnac, Y., Baudot, P., Chavane, F., Lorenceau, J., Marre, O., Monier, C., Pananceau, M., Carelli, P., Sadoc, G.: Multiscale functional imaging in V1 and cortical correlates of apparent motion. In: Masson, G., Ilg, U. (eds.) Dynamics of Visual Motion Processing, pp. 73-94. Springer (2010)

11. Albus, K.: A quantitative study of the projection area of the central and the paracentral visual field in area 17 of the cat. I. The precision of the topography. Exp. Brain Res. 24, 159-179 (1975)

12. Tootell, R.B., Silverman, M.S., Switkes, E., De Valois, R.L.: Deoxyglucose analysis of retinotopic organization in primate striate cortex. Science 218, 902-904 (1982)

13. Kalatsky, V.A., Stryker, M.P.: New paradigm for optical imaging: temporally encoded maps of intrinsic signal. Neuron 38(4), 529-545 (2003)

14. Basole, A., White, L.E., Fitzpatrick, D.: Mapping multiple features in the population response of visual cortex. Nature 423, 986-990 (2003)

15. Warnking, J., Dojat, M., Guerin-Dugue, A., Delon-Martin, C., Olympieff, S., Richard, N., Chehikian, A., Segebarth, C.: FMRI retinotopic mapping-step by step. Neuroimage 17, 1665-1683 (2002)

16. Mitchison, G., Crick, F.: Long axons within the striate cortex: their distribution, orientation, and patterns of connection. Proc. Natl. Acad. Sci. U S A. 79, 3661-3665 (1982)

17. Schwartz, E.L.: Spatial mapping in the primate sensory projection: analytic structure and relevance to perception. Biol. Cybern. 25, 181-194 (1977)

18. Moore, C.I., Nelson, S.B.: Spatio-temporal subthreshold receptive fields in the vibrissa representation of rat primary somatosensory cortex. J.Neurophysiol. 80, 2882-2892 (1998)

19. Nowak, L.G., Bullier, J.: The timing of information transfer in the visual system. In: Rockland, K.S., Kaas, J.H., Peters, A. (eds.) Extrastriate Visual Cortex in Primates, pp. 205-241. Plenum Press, New York (1997)

20. Hoffman, K.P., Stone, J.: Conduction velocity of afferents to cat visual cortex: a correlation with cortical receptive field properties. Brain Res. 32, 460-466 (1971)

21. Hirsch, J.A., Gilbert, C.D.: Synaptic physiology of horizontal connections in the cat's visual cortex. J. Neurosci. 11, 1800-1809 (1991)

22. Nauhaus, I., Busse, L., Carandini, M., Ringach, D.L.: Stimulus contrast modulates functional connectivity in visual cortex. Nature Neurosci. 12, 70-76 (2009)

23. Shoham, D., Glaser, D.E., Arieli, A., Kenet, T., Wijnbergen, C., Toledo, Y., Hildesheim, R., Grinvald, A.: Imaging cortical dynamics at high spatial and temporal resolution with novel blue voltage-sensitive dyes. Neuron 24(4), 791-802 (1999)

24. Roland, P.E.: Dynamic depolarisation fields in the cerebral cortex. Trends Neurosci. 25, 183-190 (2002)

25. Grinvald, A., Lieke, E.E., Frostig, R.D., Hildesheim, R.: Cortical point-spread function and long-range lateral interactions revealed by real-time optical imaging of macaque monkey primary visual cortex. J. Neurosci. 14, 2545-2568 (1994)

26. Jancke, D., Chavane, F., Naaman, S., Grinvald, A.: Imaging cortical correlates of illusion in early visual cortex. Nature 428, 423-426 (2004)

27. Xu, W., Huang, X., Takagaki, K., Wu, J.Y.: Compression and reflection of visually evoked cortical waves. Neuron 55(1), 119-129 (2007) 
28. Roland, P.E., Hanazawa, A., Undeman, C., Eriksson, D., Tompa, T., Nakamura, H., Valentiniene, S., Ahmed, B.: Cortical feedback depolarization waves: a mechanism of topdown influence on early visual areas. Proc. Natl. Acad. Sci. USA 103(33), 12586-12591 (2006)

29. Ahmed, B., Hanazawa, A., Undeman, C., Eriksson, D., Valentiniene, S., Roland, P.E.: Cortical dynamics subserving visual apparent motion. Cerebral Cortex 18(12), 2796-2810 (2008)

30. Benucci, A., Frazor, R.A., Carandini, M.: Standing waves and traveling waves distinguish two circuits in visual cortex. Neuron 55(1), 103-117 (2007)

31. Frégnac, Y.R., Blatow, M., Changeux, J.P., DeFelipe, J., Markram, H., Lansner, A., Maass, W., Markram, H., McCormick, D., Michel, C.M., Monyer, H., Szathmáry, E., Yuste, R.: Ups and downs in the genesis of cortical computation. In: Grillner, S., et al. (eds.) Microcircuits: The Interface between Neurons and Global Brain Function Microcircuits: Dahlem Workshop Report 93, pp. 397-437. The MIT Press, Cambridge (2006)

32. Tanifuji, M., Sugiyama, T., Murase, K.: Horizontal propagation of excitation in rat visual cortical slices revealed by optical imaging. Science 266(5187), 1057-1059 (1994)

33. Ts'o, D.Y., Gilbert, C.D., Wiesel, T.N.: Relationships between horizontal interactions and functional architecture in cat striate cortex as revealed by cross-correlation analysis. J. Neurosci. 6, 1160-1170 (1986)

34. Wertheimer, M.: Experimentelle Studien über das Sehen von Beuegung. Zeitschrift für Psychologie und Physiologie der Sinnesorgane 61, 161-265 (1912)

35. Koffka, K.: Principles of Gestalt Psychology. Harcourt Brace Javanovich, NewYork (1935)

36. Sèries, P., Georges, S., Lorenceau, J., Frégnac, Y.: Orientation dependent modulation of apparent speed: a model based on the dynamics of feed-forward and horizontal connectivity in V1 cortex. Vision Res. 42, 2781-2797 (2002)

37. Georges, S., Sèries, P., Frégnac, Y., Lorenceau, J.: Orientation dependent modulation of apparent speed: psychophysical evidence. Vision Res. 42, 2757-2772 (2002)

38. Field, D.J., Hayes, A., Hess, R.F.: Contour integration by the human visual system: evidence for a local "association field". Vision Res. 33, 173-193 (1993)

39. Hess, R., Field, D.: Integration of contours: new insights. Trends in Cognitive Sciences 3(12), 480-486 (1999) 